\title{
Overlap of Erosive and Non-erosive Reflux Diseases With Functional Gastrointestinal Disorders According to Rome III Criteria
}

\author{
Young Wook Noh, MD, Hye-Kyung Jung, MD*, Seong-Eun Kim, MD, and Sung-Ae Jung, MD \\ Department of Internal Medicine, Ewha Medical Research Institute, Ewha Womans University School of Medicine, Seoul, Korea
}

\section{Background/Aims}

Gastroesophageal reflux disease (GERD) is increasing in Asian countries. Functional dyspepsia (FD) or irritable bowel syndrome (IBS) are also prevalent and commonly overlapped with GERD. This study was conducted to compare the proportion and risk factors for overlapping reflux esophagitis (RE) and non-erosive reflux disease (NERD) with functional gastrointestinal disorders (FGIDs).

\section{Methods}

A total of 2,388 [male, 55.9\%; mean age ( \pm SD), 43.2 years $( \pm 8.4)$ ] Korean subjects who underwent the upper endoscopy for health screening were prospectively included. The subjects were asked about demographic, medical and social history by using a structured questionnaire, and FD and IBS were assessed according to the Rome III criteria.

Results

The subjects with RE were $286(12.0 \%$, male $88.5 \%, 42.8$ years) and 74 subjects had NERD (3.1\%) while the prevalence of FD and IBS were $8.1 \%$ and $10.1 \%$, respectively. The proportion of FD and IBS in NERD was higher than that of RE $(74.3 \%$ vs. $10.5 \%, p=0.000 ; 41.9 \%$ vs. $11.2 \%, p=0.000$, respectively). The epigastric pain syndrome (EPS) was more prevalent than postprandial distress syndrome in NERD. According to multiple regression analysis, high somatization score and the presence of FD increased the odd ratio for NERD. However, male gender and current smoker were significant risk factors for RE.

\section{Conclusions}

Compared to RE, NERD is more frequently overlapped with FD, especially EPS, and also are associated with significantly increased frequency of IBS. Our data draws attention to the possibility of subgrouping FGIDs and GERD to be important in understanding the pathophysiology of these conditions.

(J Neurogastroenterol Motil 2010;16:148-156)

\section{Key Words}

Gastroesophageal reflux, Functional gastrointestinal disorders, Dyspepsia, Irritable bowel syndromes

Received: December 17th, 2009 Accepted: February 23rd, 2010

(c) This is an Open Access article distributed under the terms of the Creative Commons Attribution Non-Commercial License (http://creativecommons. org/licenses/by-nc/3.0) which permits unrestricted non-commercial use, distribution, and reproduction in any medium, provided the original work is properly cited.

*Correspondence: Hye-Kyung Jung, MD

Department of Internal Medicine, Ewha Womans University Mokdong Hospital, 911-1 Mok-dong, Yangcheon-gu, Seoul 158-710, Korea

Tel: +82-2-2650-2874, Fax: +82-2-2655-2874, E-mail: junghk@ewha.ac.kr

Financial support: This work was supported by grant from academic forum of the foundation of Ewha Womans University Alumni Association. Conflicts of interest: None. 


\section{Introduction}

Cases of gastro-esophageal reflux disease (GERD), one of the most common gastrointestinal disorders in Western contries, is incresing in Asia. ${ }^{1-4}$ Over $50-70 \%$ of reflux patients have typical reflux symptoms in the absence of endoscopic mucosal breaks and are classified as non-erosive reflux disease (NERD). Functional gastrointestinal disorders (FGIDs), such as functional dyspepsia (FD) or irritable bowel syndrome (IBS) are very common in over $20 \%$ of the general population. Epidemiologic studies in Korea have demonstrated the prevalence of FD and IBS to be around 9.5-22.3\% and 2.2-9.6\%, respectively. ${ }^{6}$ Therefore, some degree of overlap between GERD and FGIDs would appear inevitable, by chance alone due to the given high prevalence rate. Indeed recent data show that patients with GERD suffer more commonly from FGIDs. ${ }^{7-9}$

Previous studies have suggested GERD to co-exist with IBS in a considerable proportion of patients. ${ }^{7,8,10,11}$ Though past definitions have included heartburn as a cardinal symptom of dyspepsia, ${ }^{12}$ it is neither necessary nor sufficient to diagnose GERD, and commonly co-occurs in patients labelled as having FD in clinical practice. ${ }^{13}$ Furthermore, it is clear that patients with epigastric symptoms would often have symptoms that arise from esophagus and low gastrointestinal tract. While Rome II classification of FGIDs categorized sub-groups by the prominent symptom, it failed to identify subgroups with homogeneous underlying pathophysiological mechanism. ${ }^{12}$ The Rome III criteria was developed to enhance research by identifying more homogeneous patients for pathophysiological study, especially FD, which was categorized into epigastric pain syndrome (EPS) and postprandial distress syndrome (PDS). ${ }^{14}$ Recent epidemologic data showed anxiety to be linked to meal-related symptoms, and not to EPS. ${ }^{15}$ The underlying mechanisms of meal-related symptoms may include fundic accommodation and visceral hypersensitivity. Whether these abnormalities are centrally mediated and hence modulated by anxiety is uncertain.

The epidemiologic study regarding the overlap between GERD and FGIDs according to Rome III criteria needs to be better defined as do risk factors, which may in turn promote more focused pathophysiological investigations.

In this study, we aimed to determine the overlap of reflux esophagitis (RE) and NERD with FGIDs according to Rome III criteria and compare the prevalence of FGIDs and its subtypes between RE and NERD. Our underlying goal was to de- termine whether distinct groups that might share a specific pathophysiology exist.

\section{Materials and Methods}

\section{Subjects}

The study population consisted of 2,932 subjects who had medical check-up for health screening and agreed to participate in this study from September 2008 to December 2008. We excluded the subjects who had significant medical illnesses $(\mathrm{n}=$ $24)$, chronic erosive gastritis $(n=287)$, peptic ulcer disease $(n=$ $223)$ and esophageal or gastric cancer $(n=5)$ by upper endoscopy, and history of major abdominal surgery, except appendectomy, cholecystectomy or hysterectomy $(\mathrm{n}=5)$.

Finally, 2,388 subjects who responded to the questionnaires and underwent upper endoscopy were included in the study. The study protocol was approved by the Institutional Review Board of Ewha Womans University.

\section{Questionnaires and laboratory test}

The original Bowel Disease Questionnaire (BDQ) was designed as a self-report instrument to measure symptoms experienced over the prior year and to collect past medical history data. ${ }^{16}$ The kappa statistic is a chance-corrected measure of agreement that represents the proportion of agreement beyond that which is expected by chance alone. Previous testing has shown the BDQ to be reliable, with a median kappa statistic for symptom items of 0.78 (range, $0.52-1.00$ ). In the outpatient setting, it has also been demonstrated to have adequate content, construct and discriminatory validity. ${ }^{17}$ The Korean BDQ, implementing the Rome III criteria, was translated from English into Korean following the proposed guideline. ${ }^{18}$ It contained 56 gastrointestinal symptom-related items; 19 items included past illness, health care use, and socio-demographic variables; and a valid measure of non-gastrointestinal somatic complaints, the Somatic Symptom Checklist (SSC). ${ }^{19}$ The modified Korean BDQ was validated with direct interview with the median kappa of 0.74 $(0.36-1.00){ }^{20}$

We also measured the severity of fatigue and stress by using Modified Brief Encounter Psychosocial Instrument (BEPSI) and fatigue severity scale (FSS), a well validated Korean version of fatigue and stress scoring questionnaires. ${ }^{21-23}$ The modified BEPSI consists of the 5 items on a 5 -point Likert type scale; a sum of 5 items was divided by 5 . A subject with a higher score ex- 
periences more stress. The internal consistency of the scale was high (Cronbach's $\alpha$ : 0.84). The FSS contains nine items developed to assess disabling fatigue and the Korean version was validated. ${ }^{22,23}$ Item responses are measured on a 7-point Likert type scale ranging from strongly disagree to strongly agree. The 9 items are combined into a total score; a lower total score indicates less effect of fatigue on everyday life. In this study, 5 out of 9 valid responses to the FSS items had to be present for the FSS total score to be calculated. If more than 5 item responses were missing, the FSS total score was set to be missing.

Glucose, triglyceride, low density lipoprotein-cholesterol (LDL-C), high density lipoprotein cholesterol (HDL-C), total cholesterol, and glucose were measured using colorimetric methods by an automatic analytical instrument (Hitachi 7600-110 Automatic analyzer, Hitachi, Tokyo, Japan).

Information about a prior risk factors included in this study is as follows:

1) Demographics: age and gender

2) Body mass index (BMI): based on height and weight which were measured by the body composition analyzer (InBody 720, BioSpace, Seoul, Korea). BMI was calculated as the individual's body weight divided by the square of height.

3) Socioeconomic status: marital status (married vs. other) and educational level (college graduate or higher was categorized as highly educated).

4) History of smoking habit and alcohol use: Smoking group was divided into current smoker and non-smoker. Alcohol use was defined by drinking alcohol more than 2-3 times a week.

\section{Definition of GERD and FGIDs}

RE was defined as endoscopic esophageal mucosal break with or without typical symptoms of GERD according to the Los Angeles classification. ${ }^{24}$ NERD was defined as having typical heartburn at least once a week without visible esophageal mucosal break on endoscopy. ${ }^{25}$ Subjects with minimal change on endoscopy with typical symptoms were classified as NERD. Subjects were classified as having IBS based upon the symptoms recorded in the questionnaire. IBS was defined by Rome III criteria. ${ }^{26}$ Definition used required recurrent abdominal pain or discomfort with at least 2 of the following characteristics: (1) relief with defecation, (2) onset associated with a change in frequency of stool, and (3) onset associated with a change in form (appearance) of stool. The subtype of IBS was based on the predominant stool pattern. IBS with diarrhea (IBS-D) was defined as having loose (mushy) or watery stools at least $25 \%$ of the time and hard stools less than $25 \%$ of bowel movements. IBS with constipation (IBS-C) was defined as having hard or lumpy stool at least $25 \%$ of the time and loose (mushy) or watery stools less than $25 \%$ of bowel movements. Mixed IBS (IBS-M) was defined as having hard or lumpy stool at least $25 \%$ of bowel movements and loose (mushy) or watery stool at least $25 \%$ of bowel movements. Unclassified IBS (IBS-U) had insufficient abnormality of stool consistency to meet the above criteria.

A criterion of FD required one or more of the following symptoms: (1) bothersome postprandial fullness, (2) early satiation, (3) epigastric pain, and (4) epigastric burning. ${ }^{14}$ No evidence of any structural diseases (including on upper endoscopy) that is likely to explain the symptom was required.

Diagnostic criteria of EPS must include all of the following: (1) pain or burning localized to the epigastrium of at least moderate severity, at least once per week, (2) the pain to be intermittent, (3) not generalized or localized to other abdominal or chest regions, (4) not relieved by defecation or passage of flatus, and (5) neither fulfilling criteria for gallbladder nor sphincter of Oddi disorders. ${ }^{14}$

Diagnostic criteria of PDS must include one or both of the following: (1) bothersome postprandial fullness, occurring after ordinary-sized meals, at least once a week and (2) early satiation that prevents finishing a regular meal, at least once a week. All the criteria fulfilled for the last 3 months with symptom onset at least 6 months prior to diagnosis.

\section{Statistical methods}

Statistical assessment was conducted using SPSS 16.0 (SPSS for Windows 16.0, SPSS Inc., Chicago, IL, USA). The continuous variables were expressed as mean $\pm \mathrm{SD}$. Statistical significance was set at $\mathrm{p}<0.05$. One-way ANOVA was used for comparison of continuous variables such as age, height, weight, waist, BMI, SSC score, FSS, stress score, and chemistry among RE, NERD, and control group. The mean values of two groups were compared using Student's $t$ test. The univariate analysis between sociodemographic features and the subtype of RE, NERD, and FGIDs were evaluated using the Chi-squared tests or the Kruskal-Wallis test. Potential predictors, including FGID and each subtypes, for RE vs. NERD were assessed using polychotomous logistic regression analyses, and adjusted by age, gender, BMI, SSC, smoking status, alcohol use, marital status, and education. The overall SSC score was included in the models to 
adjust for the tendency to over-report symptoms. The odd ratio $(\mathrm{OR})$ and $95 \%$ confidence intervals $(\mathrm{CIs})$ were computed from the estimated coefficients in the regression models.

\section{Results}

\section{Demographic and clinical characteristics of patients with GERD}

A total of 2,388 subjects were included in this study. 55.9\% of the respondents were male with mean age $( \pm \mathrm{SD})$ of $43.2( \pm 8.4)$ years. RE was found in $286(12.0 \%)$ and $88.5 \%$ of RE were male; 249 (87.1\% of RE subject) were diagnosed as Los Angeles (LA) classificaton A, 35 as LA classification B, 2 as LA classification C, and none had LA classification D. The number of patients with NERD was 74 subjects $(3.1 \%)$ with mean age of 43.8 ( \pm 8.5 ) years. Reference group meant non-RE, non-NERD group ( $\mathrm{n}=$ 2,028). There was no significant difference of age among these groups. However, there was significant association of gender among these groups (Table 1). The male predominance was noted in patients with RE and female predominance was noted in NERD compared to the reference group. BMI, the proportion of current smoker and alcohol user, serum cholesterol level, serum triglyceride level, and glucose level in the RE group were higher than these in the NERD and reference group. SSC score in NERD was greater than these in RE and reference group. In addition, headache, insomnia, fatigue, dizziness, nervousness, and hot or cold spells score in NERD were greater than these in RE and reference group. There were no significant associations FSS, BEPSI, high education, and marital status among the three groups.

\section{Prevalence of FGID and its subtype according to Rome III in RE and NERD}

The prevalence of FD was $8.1 \%$ and IBS was $10.1 \%$. The proportion of FD was higher in NERD than RE (74.3\% vs. $10.5 \%, \mathrm{p}=0.000)$. ESP and PDS were more significantly overlapped with NERD than RE, with EPS being more prevalent than PDS in NERD (68.9\% vs. $48.6 \%$, p < 0.05) (Fig. 1).

Table 1. Comparison of Clinical Characteristics Between Subjects With Non-erosive Reflux Disease and Reflux Esophagitis

\begin{tabular}{lccc}
\hline \multicolumn{1}{c}{$\begin{array}{c}\text { Variables } \\
(\mathrm{N}=2,388)\end{array}$} & $\begin{array}{c}\mathrm{RE} \\
(\mathrm{n}=286)\end{array}$ & $\begin{array}{c}\text { NERD } \\
(\mathrm{n}=74)\end{array}$ & $\begin{array}{c}\text { Reference }^{\mathrm{a}} \\
(\mathrm{n}=2,028)\end{array}$ \\
\hline Age $( \pm \mathrm{SD})(\mathrm{yr})$ & $42.8( \pm 8.4)$ & $43.8( \pm 8.5)$ & $42.7( \pm 8.8)$ \\
Male $(\%)$ & 88.5 & 47.3 & 51.6 \\
BMI $\left(\mathrm{kg} / \mathrm{m}^{2}\right)( \pm \mathrm{SD})$ & $25.1( \pm 2.8)$ & $23.9( \pm 3.4)$ & $23.5( \pm 3.1)$ \\
Waist $(\mathrm{cm})( \pm \mathrm{SD})$ & $83.5( \pm 8.8)$ & $78.8( \pm 10.0)$ & 26.9 \\
Current smoker $(\%)$ & 57.5 & 25.0 & 30.0 \\
Alcohol use $(\%)$ & 52.4 & 31.6 & 82.4 \\
High education $(\%)$ & 74.7 & 90.0 & 83.7 \\
Married $(\%)$ & 87.3 & 90.0 & $0.5( \pm 0.4)$ \\
SSC score $( \pm \mathrm{SD})$ & $0.5( \pm 0.5)$ & $0.9( \pm 0.7)$ & $2.8( \pm 1.3)$ \\
FSS $( \pm \mathrm{SD})$ & $2.9( \pm 1.2)$ & $3.3( \pm 1.2)$ & $1.8( \pm 0.7)$ \\
BEPSI score $( \pm \mathrm{SD})$ & $1.8( \pm 0.7)$ & $1.9( \pm 0.7)$ & 0.6 \\
Headache & 0.7 & 0.9 & 0.5 \\
Insomnia & 0.5 & 0.9 & 1.2 \\
Fatigue & 1.3 & 1.7 & 0.5 \\
Dizziness & 0.5 & 1.0 & 0.5 \\
Nervousness & 0.7 & 1.0 & 0.4 \\
Hot or cold spell & 0.6 & 0.9 & 193.1 \\
Cholesterol $(\mathrm{mg} / \mathrm{dL})$ & 202.3 & 191.5 & 109.8 \\
Triglyceride $(\mathrm{mg} / \mathrm{dL})$ & 145.0 & 110.0 & 116.3 \\
LDL-C $(\mathrm{mg} / \mathrm{dL})$ & 125.4 & 114.5 & 57.2 \\
HDL-C $(\mathrm{mg} / \mathrm{dL})$ & 53.8 & 56.8 & 91.0 \\
Glucose $(\mathrm{mg} / \mathrm{dL})$ & 95.5 & 91.8 & $14(0.7 \%)$ \\
Hiatal hernia $(\mathrm{n})$ & $8(2.8 \%)$ & $0(0 \%)$ &
\end{tabular}

${ }^{\mathrm{a}}$ Reference group means non-RE or non-NERD group, ${ }^{\mathrm{b}}$ High education means graduation of college or above.

RE, reflux esophagitis; NERD, non-erosive reflux disease; BMI, body mass index; SSC, somatization symptom checklist; FSS, fatigue severity score; BEPSI, brief encounter psychosocial instrument; LDL-C, low density lipoprotein-cholesterol; HDL-C, high density lipoprotein-cholesterol. 


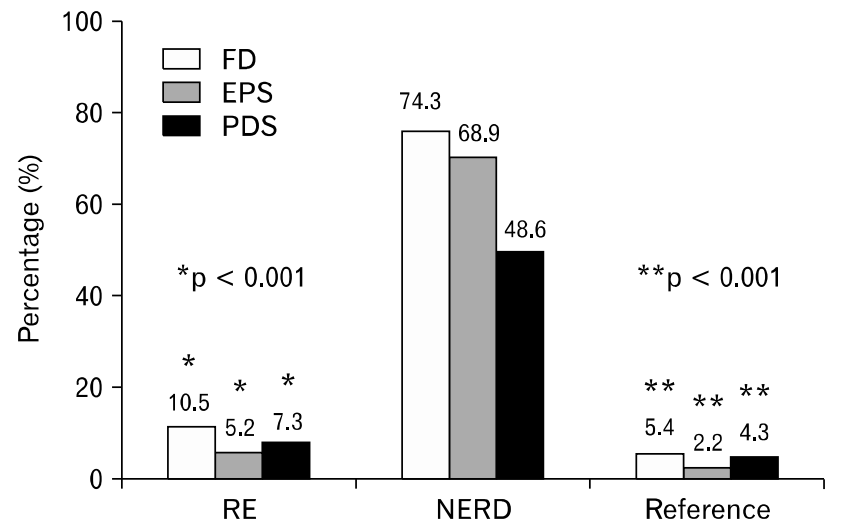

Figure 1. The proportion of functional dyspepsia (FD) and its subtypes are compared among reflux esophagitis (RE), non-erosive reflux disease (NERD), and the reference group. ${ }^{*} \mathrm{RE}$ vs. NERD, **NERD vs. Reference. EPS, epigastric pain syndrome; PDS, postprandial distress syndrome.

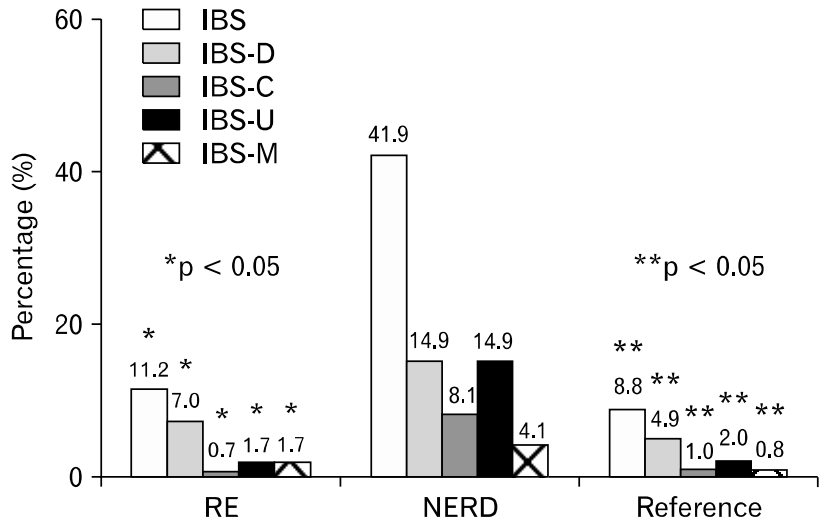

Figure 2. The proportion of irritable bowel syndrome (IBS) and its subtypes are compared among reflux esophagitis (RE), non-erosive reflux disease (NERD), and the reference group. ${ }^{*} \mathrm{RE}$ vs. NERD, **NERD vs. Reference. IBS-C, constipation-predominant IBS; IBS-D, diarrhea-predominant IBS; IBS-M, mixed pattern of IBS; IBS-U, unclassified pattern of IBS.

Table 2. Univariate and Multivariate Analysis for Predictors of Reflux Esophagitis

\begin{tabular}{|c|c|c|c|c|c|c|}
\hline \multirow{2}{*}{ Variable } & \multicolumn{3}{|c|}{ Univariate } & \multicolumn{3}{|c|}{ Multivariate } \\
\hline & OR & $95 \% \mathrm{CI}$ & p-value & OR & $95 \% \mathrm{CI}$ & $\mathrm{p}$-value \\
\hline Male sex & 7.2 & $5.00-10.50$ & 0.000 & 8.8 & $2.50-30.30$ & 0.001 \\
\hline BMI & 2.3 & $1.80-3.00$ & 0.000 & 1.1 & $1.00-1.20$ & 0.051 \\
\hline Hiatal hernia & 4.3 & $1.80-10.30$ & 0.001 & 2.9 & $0.60-14.80$ & 0.212 \\
\hline Current smoker & 3.7 & $2.30-6.00$ & 0.000 & 1.7 & $1.20-2.60$ & 0.008 \\
\hline Alcohol use & 2.6 & $1.60-4.10$ & 0.000 & 1.1 & $0.70-2.00$ & 0.634 \\
\hline $\mathrm{FD}$ & 1.4 & $0.90-2.10$ & 0.120 & & & \\
\hline EPS & 1.2 & $0.70-2.10$ & 0.583 & & & \\
\hline PDS & 1.3 & $0.80-2.00$ & 0.339 & & & \\
\hline IBS & 1.1 & $0.80-1.70$ & 0.512 & & & \\
\hline Waist & 1.1 & $1.04-1.10$ & 0.000 & & & \\
\hline HDL-C & 1.0 & $0.97-0.99$ & 0.000 & & & \\
\hline Triglyceride & 1.0 & $1.00-1.01$ & 0.000 & & & \\
\hline Glucose & 1.0 & $1.01-1.02$ & 0.000 & & & \\
\hline
\end{tabular}

OR, odd ratio; CI, confidence interval; BMI, body mass index; FD, functional dyspepsia; EPS, epigastric pain syndrome; PDS, postprandial distress syndrome; IBS, irritable bowel syndrome; HDL-C, high density lipoprotein-cholesterol.

Bold style indicates statistical significance.

The most prevalent subtype was IBS-D $(\mathrm{n}=131,54.4 \%)$ while the number of subjects with IBS-C and IBS-M was 29 $(12.0 \%)$ and $25(10.4 \%)$, respectively. Overlap of IBS and NERD was significantly more frequent than with RE (41.9\% vs. $11.2 \%, \mathrm{p}=0.000)$ and the reference group $(41.9 \%$ vs. $8.8 \%, \mathrm{p}$ $=0.000)$, however, there was no difference between RE and the reference group. Overlap of all categories of IBS subtypes was found more frequently in NERD than in RE and the reference group (p < 0.05) (Fig. 2).

\section{Assessment of predictors for RE and NERD by} univariate and multivariate analyses

In univariated analysis, male sex, BMI, hiatal hernia, current smoking, alcohol user, waist circumference, low HDL-cholesterol level, high cholesterol, triglyceride, and glucose level were significant predictors for RE. The significant independent predictors of $\mathrm{RE}$ in multivariate analysis were male sex (OR, 8.8; 95\% CI, 2.5-30.3; $\mathrm{p}=0.001)$ and current smoker (OR, 1.7; 
95\% CI, 1.2-2.6; $\mathrm{p}=0.008)($ Table 2).

In univariate analysis, SSC score, insomnia, nervousness, IBS, FD, and each subtype were statistically associated with NERD. However in multivariate analysis, significant predictors of NERD were SSC score (OR, 2.7; 95\% CI, 1.03-7.10; p = $0.043)$ and $\mathrm{FD}(\mathrm{OR}, 56.4$; 95\% CI, 19.8-160.5; p = 0.000). EPS was a very strong predictor for NERD (OR, 82.4; 95\% CI, 25.5-266.7; $\mathrm{p}=0.000)$, however, PDS, IBS, and subtype were not related with NERD in multivariate analysis (Table 3 ).

\section{Discussion}

The morphologic spectrum of esophageal involvement in GERD encompasses NERD, erosive reflux disease, and Barrett's esophagus based on endoscopic findings. However, there is still no consensus on whether GERD represents one disease that can progress from NERD to RE and Barrett's esophagus, or whether it is a spectrum of different conditions with its own clinical, pathophysiologic, and endoscopic characteristics. Moreover, the development of new instruments and techniques, e.g., magnifying endoscopy, have made these arguments less useful. ${ }^{27}$ However, studies evaluating the epidemiology, pathophysiology, and treatment of GERD commonly assessed RE and
NERD as separate groups. ${ }^{28}$

In the present study, RE and NERD, the 2 main phenotypes of GERD, seem to have distinct clinical characteristics and risk factors. Male sex and smoking were found to be the prime risk factors for RE, while a predictor for NERD was high somatization score. NERD group was more overlapped with FD and IBS than $\mathrm{RE}$ and the reference group.

Both GERD and FD are recognized as the most common chronic diseases of adults in developed countries. Since both have high prevalence in the community, some overlap should be expected. The previous studies reported the prevalence of overlap of GERD with dyspepsia around 7.5-8.4\%. ${ }^{7,29}$ Similarly, in the present study, prevalence of FD was more prevalent in GERD, especially NERD, compared with the reference group. IBS was found to be more frequently overlapped with NERD than with RE. However, we noticed IBS to be equally common in both RE and the reference group. The previous study reported the prevalence rates of IBS in GERD to range from 19 to $71 \%{ }^{30}$ In contrast, the rate of IBS in the non-GERD community was only around $5 \%$. $^{30}$

We demonstrated significant overlap among NERD, FD, IBS, and its subtypes. However, the demonstration of significant overlap of symptoms raised the question of whether the FGIDs

Table 3. Univariate and Multivariate Analysis for Predictors of Non-erosive Reflux Disease

\begin{tabular}{|c|c|c|c|c|c|c|c|c|c|}
\hline \multirow{2}{*}{ Variable } & \multicolumn{3}{|c|}{ Univariate } & \multicolumn{6}{|c|}{ Multivariate } \\
\hline & OR & $95 \% \mathrm{CI}$ & $\mathrm{p}$-value & OR & $95 \% \mathrm{CI}$ & p-value & OR & $95 \% \mathrm{CI}$ & $\mathrm{p}$-value \\
\hline Male sex & 0.7 & $0.40-1.10$ & 0.132 & 0.7 & $0.3-1.8$ & 0.428 & 0.5 & $0.14-1.40$ & 0.179 \\
\hline BMI & 1.2 & $0.70-1.90$ & 0.550 & 1.0 & $0.4-2.8$ & 0.973 & 0.8 & $0.30-2.50$ & 0.688 \\
\hline SSC score & 4.3 & $2.40-7.70$ & 0.000 & 1.9 & $0.9-4.5$ & 0.099 & 2.7 & $1.03-7.10$ & 0.043 \\
\hline $\mathrm{FD}$ & 45.3 & $26.20-78.40$ & 0.000 & 56.4 & $19.8-160.5$ & 0.000 & & & \\
\hline EPS & 84.7 & $48.60-147.80$ & 0.000 & & & & 82.4 & $25.50-266.70$ & 0.000 \\
\hline PDS & 19.2 & $11.80-31.40$ & 0.000 & & & & 2.2 & $0.70-7.10$ & 0.185 \\
\hline IBS-C & 8.8 & $3.50-22.30$ & 0.000 & & & & & & \\
\hline IBS-D & 3.2 & $1.60-6.20$ & 0.001 & & & & & & \\
\hline IBS-M & 4.4 & $1.20-15.10$ & 0.018 & & & & & & \\
\hline IBS-U & 8.8 & $4.40-17.80$ & 0.000 & & & & & & \\
\hline Nervousness & 1.6 & $1.10-2.30$ & 0.007 & & & & & & \\
\hline Hiatal hernia & 0.0 & $0.00-0.00$ & 0.998 & & & & & & \\
\hline Alcohol use & 0.9 & $0.40-2.50$ & 0.873 & & & & & & \\
\hline Current smoker & 0.7 & $0.30-2.00$ & 0.537 & & & & & & \\
\hline
\end{tabular}

OR, odd ratio; CI, confidence interval; BMI, body mass index; SSC, somatization symptom checklist; FD, functional dyspepsia; IBS, irritable bowel syndrome; EPS, epigastric pain syndrome; PDS, postprandial distress syndrome; IBS-C, constipation-predominant IBS; IBS-D, diarrhea-predominant IBS; IBS-M, mixed pattern of IBS; IBS-U, unclassified pattern of IBS.

Bold style indicates statistical significance. 
should be considered as multiple separate disorders or more as of a common clinical entity.

There lies a significant interest in the pathophysiology of NERD. Chemosensitivity may explain the heartburn in NERD. In animal and human studies, dilatation of the intracellular spaces may explain heartburn in patients with NERD within physiological acid exposure. ${ }^{31,32}$ The increased paracellular permeability may irritate the afferent nerve in the esophageal epithelium. It is also possible that visceral hypersensitivity to be an important pathophysiologic mechanism in IBS and FD. ${ }^{33-35}$ Stress and psychological comorbidity seem also to be another factors in the generation of symptoms in patients with NERD through brain gut interaction. ${ }^{36}$ Pogromov et al. ${ }^{37}$ analyzed the psycho-autonomic aspects in patients with GERD and found that patients with NERD were characterized by more pronounced emotional, motivational, and autonomic disorders compared to RE patients. The present study documented the somatization score as a significant predictor for NERD, but not for RE. The self-reported insomnia and nervousness are significantly related with NERD and these findings are consistent with the previous study. Fass et al. $^{38}$ showed $68.0 \%$ of FD, $71.2 \%$ of overlap of IBS-FD, and $50.2 \%$ of IBS to have sleep disturbances in population based study. Dysregulation of brain-gut interaction has been suggested as an important factor in the pathogenesis of IBS. ${ }^{39}$ Especially the diarrhea-predominant IBS was related with autonomic nervous dysfunction, but independent with psychological disturbance. ${ }^{39}$ Putative brain gut interaction may involve in altered sleep, GERD, and FGID. ${ }^{40}$

Factor analyses have demonstrated some of gastrointestinal symptoms to occur in a cluster suggesting a specific pathophysiology. Camilleri et al. ${ }^{41}$ reported the factor analysis which categorized GI symptoms into groups reflecting GERD and dyspepsia with the meal-related symptom cluster. In the present study, EPS is a strongest predictor for NERD, not PDS. In clinical practice, distinguishing GERD from FD may be difficult. ${ }^{42}$ Studies have established that clinicians often fail to recognize typical reflux symptoms. Furthermore, GERD may mimic other conditions including FD. ${ }^{43}$ The previous study presented that one quarter of FD without reflux symptoms had pathological acid exposure on the distal esophagus. ${ }^{13}$ And, epigastric pain was more prevalent in dyspeptic patients with pathologic esophageal $\mathrm{pH}$ monitoring. Epigastric pain was a prominent symptom in dyspeptic patients who were likely to respond to proton pump inhibitors. ${ }^{44}$ However, it is controversial whether these group could be defined as the subtypes of FD.
However, there is a concern regarding the instability of subtypes of FGIDs or FD subtypes. Rome II criteria failed to find major differences in natural histories among FD subtypes. ${ }^{36}$ Defining a clear distinction of these overlapping group between NERD and FGIDs may be important in determining the management. Their clinical characteristics may belong in the spectrum of FGIDs more appropriately than in that of GERD. This separation might spare these intractable patients from wasteful courses of potent acid suppression or surgical options. ${ }^{45}$

Our study has several strengths and limitations. This study enrolled a large sample of 2,388 study participants who underwent upper endoscopy and validated questionnaires in health promotion center. Therefore, we were able to ascertain how many had objective evidences of reflux disease and FD. This is our strong point since there are substantial proportions of organic disease, such as stomach cancer or Helicobacter pylori related disease in Far East Asia. However, there is a possibility of sampling bias, such as volunteer bias. Two third of the study subjects from Health Promotion Center were offered with a medical examination at workplace as a reward and only $1 / 3$ paid by themselves. Therefore, some proportion of subjects who were enrolled in Health Promotion Center had different exposures from general population. The previous study in Korea demonstrated health screening population have higher income and educational status compared with general Korean population. However, other factor such as BMI, smoking, alcohol habit, or co-morbidities were comparable to the background population. ${ }^{44,46}$ These factors were not significantly different among RE, NERD, and the reference group in our study.

In conclusion, NERD is more frequently overlapped with FD than RE, especially with EPS, and presents with significantly increased frequency of IBS. This data draws attention to the possibility that subgrouping the FGIDs and GERD to be important in understanding the pathophysiology and management of these conditions.

\section{References}

1. Dent J, Brun J, Fendrick A, et al. An evidence-based appraisal of reflux disease management - the Genval Workshop Report. Gut 1999;44(suppl 2):S1-S16.

2. Locke GR 3rd, Talley NJ, Fett SL, Zinsmeister AR, Melton LJ 3rd. Prevalence and clinical spectrum of gastroesophageal reflux: a population-based study in Olmsted County, Minnesota. Gastroenterology 1997;112:1448-1456.

3. Agréus L, Svärdsudd K, Talley NJ, Jones MP, Tibblin G. Natural 
history of gastroesophageal reflux disease and functional abdominal disorders: a population-based study. Am J Gastroenterol 2001;96: 2905-2914

4. Fock KM, Talley NJ, Fass R, et al. Asia-Pacific consensus on the management of gastroesophageal reflux disease: update. J Gastroenterol Hepatol 2008;23:8-22.

5. Dent J. Gastro-oesophageal reflux disease. Digestion 1998;59: 433-445.

6. Lee SY, Lee KJ, Kim SJ, Cho SW. Prevalence and risk factors for overlaps between gastroesophageal reflux disease, dyspepsia, and irritable bowel syndrome: a population-based study. Digestion 2009; 79:196-201.

7. Locke GR 3rd, Zinsmeister AR, Fett SL, Melton LJ 3rd, Talley NJ. Overlap of gastrointestinal symptom complexes in a US community. Neurogastroenterol Motil 2005;17:29-34.

8. Jung HK, Halder S, McNally $M$, et al. Overlap of gastro-oesophageal reflux disease and irritable bowel syndrome: prevalence and risk factors in the general population. Aliment Pharmacol Ther 2007;26:453-461.

9. Talley NJ, Ruff K, Jiang X, Jung HK. The Rome III Classification of dyspepsia: will it help research? Dig Dis 2008;26:203-209.

10. Talley NJ. Overlapping abdominal symptoms: why do GERD and IBS often coexist? Drugs Today 2006;42(suppl B):3-8.

11. Pimentel M, Rossi F, Chow EJ, et al. Increased prevalence of irritable bowel syndrome in patients with gastroesophageal reflux. J Clin Gastroenterol 2002;34:221-224.

12. Karamanolis G, Caenepeel P, Arts J, Tack J. Association of the predominant symptom with clinical characteristics and pathophysiological mechanisms in functional dyspepsia. Gastroenterology 2006; 130:296-303.

13. Tack J, Caenepeel P, Arts J, Lee KJ, Sifrim D, Janssens J. Prevalence of acid reflux in functional dyspepsia and its association with symptom profile. Gut 2005;54:1370-1376.

14. Tack J, Talley NJ, Camilleri M, et al. Functional gastroduodenal disorders. Gastroenterology 2006;130:1466-1479.

15. Aro $\mathbf{P}$, Talley NJ, Ronkainen J, et al. Anxiety is associated with uninvestigated and functional dyspepsia (Rome III criteria) in a Swedish population-based study. Gastroenterology 2009;137:94-100.

16. Talley NJ, Phillips SF, Melton LJ 3rd, Wiltgen C, Zinsmeister AR. A patient questionnaire to identify bowel disease. Ann Intern Med 1989;111:671-674.

17. Talley NJ, Phillips SF, Wiltgen CM, Zinsmeister AR, Melton LJ $3 \mathrm{rd}$. Assessment of functional gastrointestinal disease: the bowel disease questionnaire. Mayo Clin Proc 1990;65:1456-1479.

18. Guillemin F, Saraux A, Fardellone P, Guggenbuhl P, Behier JM, Coste J. Detection of cases of inflammatory rheumatic disorders: performance of a telephone questionnaire designed for use by patient interviewers. Ann Rheum Dis 2003;62:957-963.

19. Attanasio V, Andrasik F, Blanchard EB, Arena JG. Psychometric properties of the SUNYA revision of the Psychosomatic Symptom Checklist. J Behav Med 1984;7:247-257.

20. Song HJ, Jung HK, Yeom HJ, et al. Reliability and validity of Korean Bowel Disease Questionnaire and prevalence of functional gastrointestinal disorders in Korea [abstract]. Gut 2009;58(suppl II):A112.

21. Yim JH, Bae JM, Choi SS, et al. The validity of modified Korean translated BEPSI as instrument of stress measurement in outpatient clinic. J Korean Acad Fam Med 1996;17:42-49.

22. Song $\mathrm{CH}$, Chung KI, Song SW, Kim KS. The effects of L-theanine containing functional beverage on mental relaxation and fatigue perception. J Korean Acad Fam Med 2002;23;637-645.

23. Chung KI, Song CH. Clinical usefulness of fatigue severity scale for patients with fatigue, and anxiety or depression. Korean J Psychosom Med 2001;9:164-173.

24. Lundell LR, Dent J, Bennett JR, et al. Endoscopic assessment of oesophagitis: clinical and functional correlates and further validation of the Los Angeles classification. Gut 1999;45:172-180.

25. Fass R, Fennerty MB, Vakil N. Nonerosive reflux disease-current concepts and dilemmas. Am J Gastroenterol 2001;96:303-314.

26. Longstreth GF, Thompson WG, Chey WD, Houghton LA, Mearin F, Spiller RC. Functional bowel disorders. Gastroenterology 2006;130:1480-1491.

27. Vakil N, van Zanten SV, Kahrilas P, Dent J, Jones R. The Montreal definition and classification of gastroesophageal reflux disease: a global evidence-based consensus. Am J Gastroenterol 2006;101: 1900-1920.

28. Fass R. Erosive esophagitis and nonerosive reflux disease (NERD): comparision of epidemiologic, physiologic, and therapeutic characteristics. J Clin Gastroenterol. 2007;41:131-137.

29. Kitaçpioğlu G, Mandiracioğlu A, Caymaz Bor C, Bor S. Overlap of symptoms of dyspepsia and gastroesophageal reflux in the community. Tur J Gastroenterol 2007;18:14-19.

30. Nastaskin I, Mehdikhani E, Conklin J, Park S, Pimentel M. Studying the overlap between IBS and GERD: a systematic review of the literature. Dig Dis Sci 2006;51:2113-2120.

31. Ferreira KT, Hill BS. The effect of low external $\mathrm{pH}$ on properties of the paracellular pathway and junctional structure in frog skin. J Physiol 1982;332:59-67.

32. Hopwood D, Milne G, Logan KR. Electron microscopic changes in human oesophageal epithelium in oesophagitis. J Pathol 1979; 129:161-167.

33. Trimble KC, Pryde A, Heading RC. Lowered oesophageal sensory thresholds in patients with symptomatic but not excess gastro-oesophageal reflux: evidence for a spectrum of visceral sensitivity in GORD. Gut 1995;37:7-12.

34. Bouin M, Plourde V, Boivin M, et al. Rectal distention testing in patients with irritable bowel syndrome: sensitivity, specificity, and predictive values of pain sensory thresholds. Gastroenterology 2002; 122:1771-1777.

35. Coffin B, Azpiroz F, Guarner F, Malagelada JR. Selective gastric hypersensitivity and reflex hyporeactivity in functional dyspepsia. Gastroenterology 1994;107:1345-1351.

36. Halder SL, Locke GR 3rd, Schleck CD, Zinsmeister AR, Melton LJ 3rd, Talley NJ. Natural history of functional gastrointestinal disorders: a 12-year longitudinal population-based study. Gastroenterology 2007;133:799-807.

37. Pogromov AP, Diukova GM, Rykova SM, Bein AM. Psycho-autonomic aspects in patients with gastroesophageal reflux disease, and functional esophageal disorders. Klin Med 2005;83:41-44.

38. Fass R, Fullerton S, Tung S, Mayer EA. Sleep disturbances in clinic patients with functional bowel disorders. Am J Gastroenterol 2000;95:1195-2000. 
39. American Gastroenterology Association. American Gastroenterological Association medical position statement: irritable bowel syndrome. Gastroenterology 2002;123:2105-2107.

40. Jung HK, Choung RS, Talley NJ. Gastroesophageal reflux disease and sleep disorders: evidence for a causal link and therapeutic implications. J Neurogastroenterol Motil 2010;16:22-29.

41. Camilleri M, Dubois D, Coulie B, et al. Prevalence and socioeconomic impact of upper gastrointestinal disorders in the United States: results of the US upper gastrointestinal study. Clin Gastroenterol Hepatol 2005;3:543-552.

42. Carlsson R, Dent J, Bolling-Sternevald E, et al. The usefulness of a structured questionnaire in the assessment of symptomatic gastroesophageal reflux disease. Scand J Gastroenterol 1998;33:1023-
1029.

43. Dent J. Definitions of reflux disease and its separation from dyspepsia. Gut 2002;50(suppl 4):iv17-iv20.

44. Kim N, Lee SW, Cho SI, et al. The prevalence of and risk factors for erosive oesophagitis and non-erosive reflux disease: a nationwide multicentre prospective study in Korea. Aliment Pharmacol Ther 2008;27:173-185.

45. Quigley EM. Functional dyspepsia (FD) and non-erosive reflux disease (NERD): overlapping or discrete entities? Best Pract Res Clin Gastroenterol 2004;18:695-706.

46. Kim JH, Hwang JK, Kim J, et al. Endoscopic findings around the gastroesophageal junction: an experience from a tertiary hospital in Korea. Korean J Intern Med 2008;23:127-133. 